\title{
Interphase Boundary Segregation into an Ordered Core-Shell Structure with a Shell Containing Two Unit-Cells
}

\section{Supplementary Information}

Hongbo Xie ${ }^{\mathrm{a}}$, Weixin Lou ${ }^{\mathrm{a}}$, Xiaobo Zhao ${ }^{\mathrm{a}}$, Junyuan Bai ${ }^{\mathrm{b}}$, Shanshan $\mathrm{Li}^{\mathrm{a}}$, Na Xiao ${ }^{\mathrm{c}}$, Hucheng Pan ${ }^{\mathrm{b}}$, Xueyong Panga, Hongxiao Lib, Yuping Ren* a, d, Gaowu Qin*b

${ }^{a}$ Key Laboratory for Anisotropy and Texture of Materials (Ministry of Education), School of Materials Science and Engineering, Northeastern University, Shenyang 110819, China

${ }^{\mathrm{b}}$ State Key Laboratory of Rolling and Automation, Northeastern University, Shenyang 110819, China

c Analytical and Testing Center, Northeastern University, Shenyang, 110819, China

d Research Center for Metal Wires, Northeastern University, Shenyang 110819, China

* Corresponding author. Email: renyp@atm.neu.edu.cn (Y. P. Ren); qingw@smm.neu.edu.cn (G. W. Qin). 


\section{Supplementary Figures}

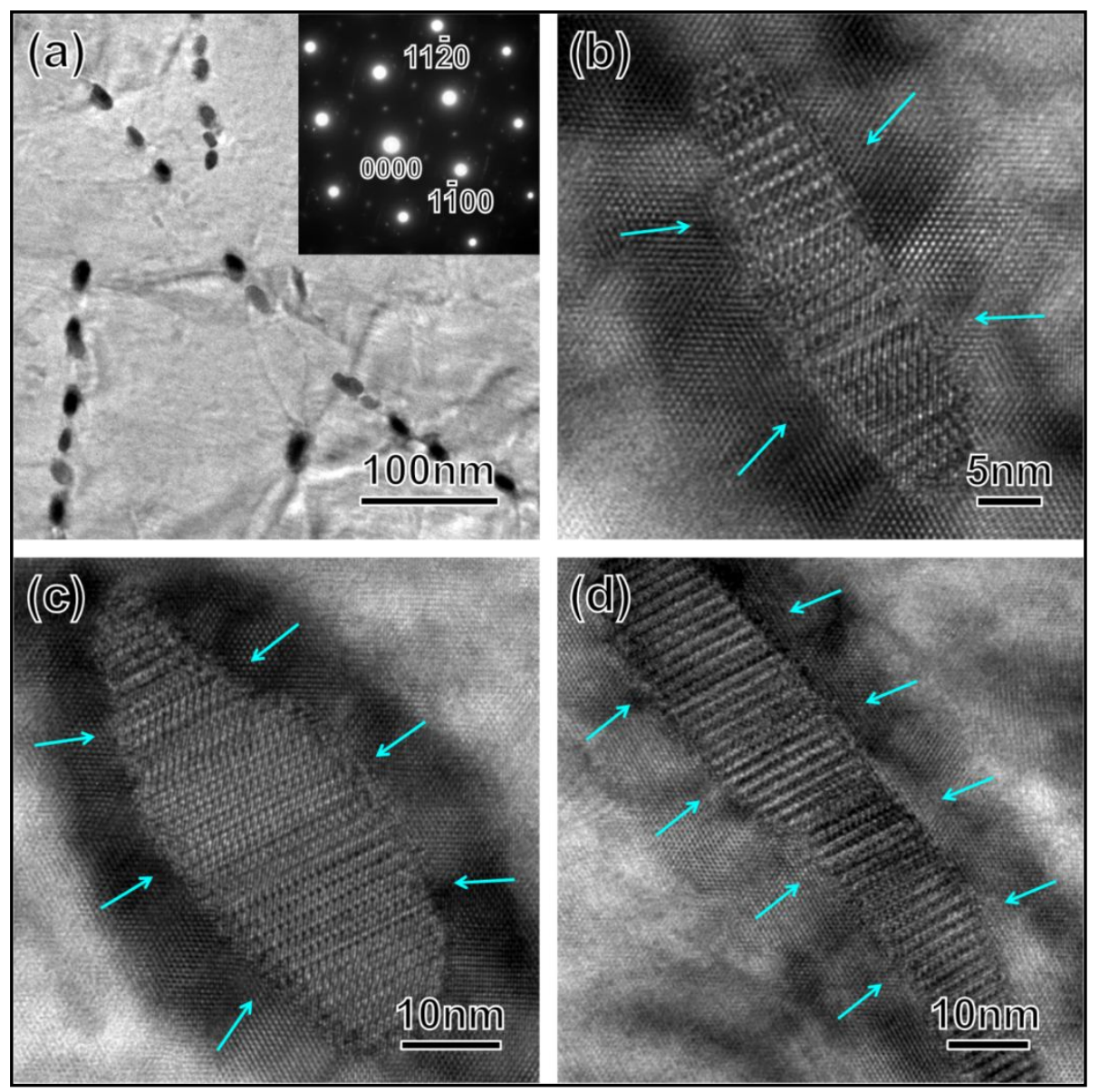

Figure S1. TEM images of the Mg-1.5In-0.5Ca alloy isothermally aged at $200{ }^{\circ} \mathrm{C}$ for $48 \mathrm{~h}$, the sample prepared by $\mathrm{Ar}$ ion milling. (a) Bright-field TEM image, and the top-right inset is the corresponding select-area electron diffraction (SAED) pattern. (b-d) High-resolution TEM images, some precipitate-particles in Supplementary Figure S1a are enlarged and shown in Supplementary Figures S1b-d. As indicated by the arrows, the shell regions between the precipitate-particles and $\alpha$-Mg matrix show the higher contrast, owing to the increase of the heavier In atoms. The electron beam is parallel to the $[0001]_{\alpha}$. 


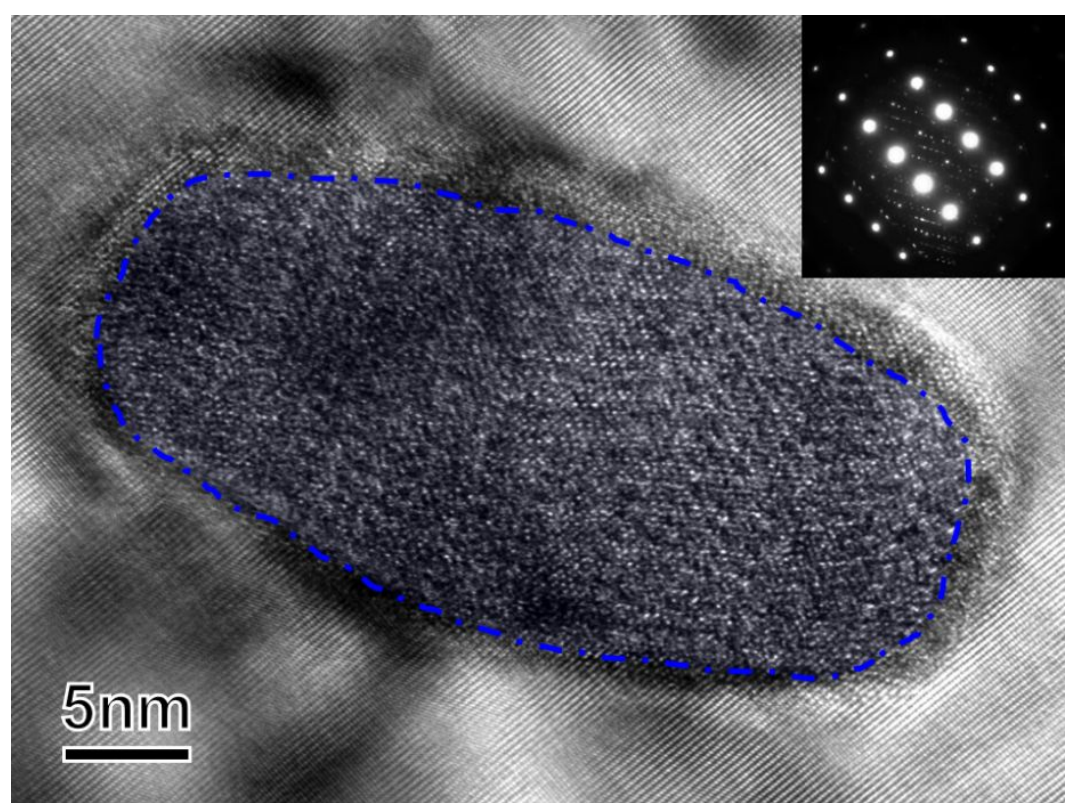

Figure S2. HRTEM image of the Mg-1.5In- $0.5 \mathrm{Ca}$ alloy isothermally aged at $200{ }^{\circ} \mathrm{C}$ for $48 \mathrm{~h}$, the sample prepared by Ar-ion milling. The top-right inset is the corresponding SAED pattern. The electron beam is parallel to the $[10 \overline{1} 0]_{\alpha}$. 


\section{Supplementary Tables}

Table S1. The formation energies and corresponding computation crystal models of the ordered hcp-Mg/In solid solutions.

\begin{tabular}{llll}
\hline \multirow{2}{*}{ Type of solid solutions } & \multicolumn{3}{c}{ Atomic models } \\
\cline { 2 - 4 } Mg-25 at.\% In (ordered) & Viewed along $[0001]_{\alpha}$ & 3D view \\
Mg-75 at.\% In (ordered) & & \\
& &
\end{tabular}


Table S2. The formation energies and corresponding computation crystal models of the disordered hcp-Mg/In solid solutions.

\begin{tabular}{|c|c|c|c|}
\hline Type of solid solutions & $3 \mathrm{D}$ views of the atomic models & Formation energies (meV) & Average values \\
\hline Mg-25 at.\% In (disordered-1) & & -48.88 & \\
\hline Mg-25 at.\% In (disordered-2) & & -48.55 & -48.80 \\
\hline Mg-25 at.\% In (disordered-3) & & -48.97 & \\
\hline Mg-75 at. $\%$ In (disordered- 1$)$ & & -21.08 & \\
\hline Mg-75 at. $\%$ In (disordered- 2$)$ & & -17.19 & -14.62 \\
\hline Mg-75 at. $\%$ In (disordered-3) & & -5.60 & \\
\hline
\end{tabular}


Table S3. The formation energies and corresponding computation crystal models of the equilirium MgIn intermetallic compounds.

\begin{tabular}{|c|c|c|c|}
\hline \multirow{2}{*}{ Intermetallic compounds } & \multicolumn{2}{|c|}{ Atomic models } & \multirow{2}{*}{ Formation energies $(\mathrm{meV})$} \\
\hline & Viewed along $[0001]_{\alpha}$ & $3 \mathrm{D}$ view & \\
\hline $\mathrm{Mg}_{3} \operatorname{In}\left(\mathrm{Ll}_{2}, \mathrm{AuCu}_{3}\right)$ & & & -85.29 \\
\hline $\operatorname{MgIn}_{3}\left(\mathrm{Ll}_{2}, \mathrm{AuCu}_{3}\right)$ & & & -45.76 \\
\hline
\end{tabular}

